\title{
Wavelet Based Simulation and Analysis of Single and Multiple Power Quality Disturbances
}

\author{
Fayyaz Jandan \\ IICT, Mehran University of Engineering Technology, \\ Jamshoro, Pakistan and Electrical Engineering Department, \\ Quaid-e-Awam University College of Engineering Science and \\ Technology, Larkana, Pakistan \\ fayyaz.jandan@quest.edu.pk \\ Zubair Ahmed Memon \\ Electrical Engineering Department, \\ Mehran University of Engineering Technology, \\ Jamshoro, Pakistan \\ zubair.memon@faculty.muet.edu.pk
}

\author{
Suhail Khokhar \\ Electrical Engineering Department, \\ Quaid-e-Awam University of Engineering Science \\ and Technology, \\ Nawabshah, Pakistan \\ suhail@quest.edu.pk \\ Syed Abid Ali Shah \\ School of Engineering and Applied Sciences, \\ Aston University, \\ Birmingham, UK \\ bukhars@aston.ac.uk
}

\begin{abstract}
Improving power quality disturbance (PQD) detection and automatic classification has been a major concern ever since the emergence of sensitive non-linear devices. The role of distributed generation in a power system is the main source of PQDs. Short-term and long-term duration single and multiple complex PQDs are difficult to monitor and need higher accuracy and time. This paper presents the analysis of different and distinctive combinations of PQDs. Variety of single and multiple PQD samples are generated using Matlab environment conferring to IEEE STD 1159-2009. Such disturbance samples are accurately detected and analyzed from waveform patterns using multi resolution analysis based discrete wavelet transform. The generation of samples and detection lies in fact that it can allow the feature extraction process for the training/testing sample features for machine learning based automatic recognition of disturbance types.
\end{abstract}

Keywords-power quality disturbances (PQDs); power quality generation; discrete wavelet transform (DWT); multi resolution analysis (MRA)

\section{INTRODUCTION}

The term power quality (PQ) has reached a vigorous standing since late 1980s [1]. It plays a vital and demanding contribution in industrial and utility operations for reliable, efficient and uninterrupted power supply. In a power system, constant technological advancement, use of fast control equipment, and non-conventional energy sources are considered pavements for potential progress, sustainable stability and economic development of any country [2]. PQD analysis is predominantly performed to evaluate power system operational failures, designing and troubleshooting of transmission lines, distribution and utilizations systems [3]. Generally PQDs are outlined as disparity in normal voltage and or current amplitude, phase angle and frequency $[4,5]$. Such disturbances are mainly engendered due to non-linear loads/devices at consumer premises, power electronic converters, short circuit faults, lightning and switching actions of solid state switching devices, induction machines, motors, power transmission lines, capacitor banks etc. [6]. Moreover, renewable energy sources integration and dispersed generation systems in existing power system infrastructure are also leading ground sources of disturbances [7]. PQDs lessen reliability, performance and working life of the electrical equipment and may also cause failures of the high voltage utility equipment (i.e. insulation degradation, failures in high-voltage and lowvoltage protection systems, false tripping by relaying systems etc.) as well as consumer's household equipment. Persistent disturbing events if not mitigated instantly, may cause complete discontinuity of the power transmission system or distribution of supply systems. PQDs are generally categorized as, magnitude variations, transients, steady state variations or harmonics. Magnitude variations are further classified as voltage/current sag (10-90\%), swell (110-180\%), interruption $(<10 \%)$ [8], whereas transients can be classified as impulsive and oscillatory. Steady-state PQDs are harmonics, flickers and notches. Multiple PQ disturbances, i.e. combination of more than one disturbances, in the power system and such operations may give rise to a variety of hybrid disturbances $[9,10]$.

PQ is a diversified area and urges amassed research. Therefore it can be broadly categorized and congregated in six aspects: concepts, effects, solutions, sources, instrumentation, modeling and analysis [11]. For the research in classification and PQ event recognition, extensive areas are: PQ standards, time domain, and transform domain wave analysis. It is known that PQDs can be reduced by the application of several types of filters and compensators [11]. In order to identify and avoid disturbances and their causes, regular monitoring of instantaneous events of voltage and current waveform parameters at the rated power frequency must be analyzed,

Corresponding author: F. Jandan 
from power generation to distribution/utilization systems [11, 12]. Substantial computational time and resources are required, since disturbance event data collection and manual monitoring lacks reliability. All types of discontinuities and disturbances are unable to be detected by most of PQ analyzers. Abnormal event occurrence can be detected by effective automatic and intelligent algorithms. The purpose of perseverance and reestablishment of normal power supply without a slight interruption for even a fraction of seconds is achieved through intelligent monitoring and analysis. PQDs detection carries a lot of concerns, including: the PQD types, the number of single and multiple disturbances under observation, the original causes of disturbances, the choice of signal processing technique etc. Moreover, for pattern recognition, feature extraction capability and choice of artificial intelligent technique according to classification performance characteristics are also considered. A variety of signal processing techniques can be used for feature extraction through PQD analysis [8, 13]. Classified techniques are Fourier transform (FT), Kalman filtering, wavelet transform (WT), Stockwell-transform (S-Transform), Hilbert-Huang transform (HHT), Gabor transform (GT) etc. [8]. PQDs are observed sometimes dynamically slow or abrupt in nature, thus timely PQD event detection is imperative for analysis and monitoring. This brings us to the application of WT. Other transforms can also be implemented (Figure 1), but issues of complexity, erroneous detection in dynamic and sudden disturbances, nonlocalization in time or space and non- suitability with dynamic signal like PQDs, make WT a robust and suitable tool for detection of event in time-frequency domain [8].

In this study, a general analysis on PQ associated with power systems is presented. Real PQ issues are simulated through single and multiple disturbance waveform patterns generation. Power system operations frequently perceive disturbances as mentioned in Table I. A number of samples for each disturbance are generated through model equations in Matlab workspace environment. Such model based generated disturbances are then passed through DWT filters. Along with the achievement of event detection in time frequency localization, feature extraction vector from approximation and detail levels for machine learning based classification can be obtained. Extracted features are used for the training and testing of classifier [14-18].

\section{WAVELET TRANSFORM}

Wavelet, a rapidly decaying wave alternation, having zero mean, extends and exists for finite duration unlike sinusoid which extends to infinity. Wavelet analysis is well suited for non-periodic signals. It may contain both stationary components and transients, such as the ones that can be found on PQDs. Some well-known wavelet types are shown in Figure 2. The availability of a wide range of derivatives of wavelets is a key strength of wavelet analysis. Daubechies 4 i.e. $d 4$, a type of Daubechies wavelet class, is mostly adopted for the analysis of PQDs because of its comparably analogous characteristics with PQ events [19]. Wavelet analysis has baseband characteristics in frequency domain. The major advantage of wavelet analysis is the varying window size, which is wide for slowly varying changes i.e. low frequencies, and narrow for abrupt changes. As a result there is optimal time-frequency localization in all frequency ranges $[20,21]$.

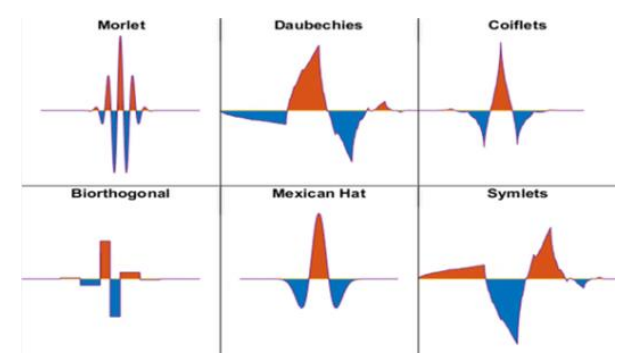

Fig. 1. Basic types of mother wavelets
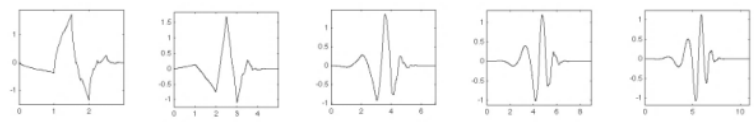

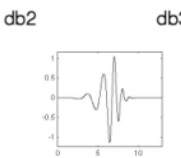

$\mathrm{db} 7$

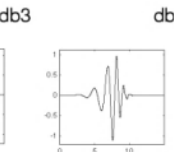

$\mathrm{db} 8$ $\mathrm{db} 4$

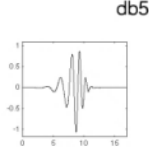

$\mathrm{db} 9$

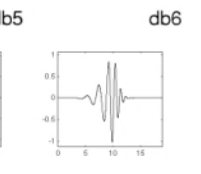

$\mathrm{db} 10$
Fig. 2. Types of Daubechies (db) wavelet

\section{CONTINUOUS VS DISCRETE WAVELET}

WT technique has a significant role in discontinuity detection for PQDs, and has also been found to be a powerful tool in the feature extraction process. In continuous WT $(\mathrm{CWT})$, scaling and translation of mother wavelet $\varphi(t)$ provide the information of time frequency resolution of the original distorted waveform signal $[1,2]$. The mathematical equation of CWT for a given disturbance signal $x(t)$ with respect to $\varphi(t)$ is given as:

$$
\operatorname{CWT}(\mathrm{c}, \mathrm{d})=\frac{1}{\sqrt{|\mathrm{c}|}} \int_{-\infty}^{\infty} \mathrm{x}(\mathrm{t}) \varphi\left(\frac{\mathrm{t}-\mathrm{d}}{\mathrm{c}}\right) \mathrm{dt}
$$

where $c$ and $d$ are real positive numbers. $c$ is the scaling factor, inversely proportional to frequency. It corresponds to signal dilation or shrinking in time domain whereas $d$ is the translation factor corresponds to wavelet shifting.

Although CWT is upright with time frequency analysis, it has some limitations when compared to discrete WT (DWT):

- CWT computation by computer simulation is discretized CWT, which is not a true discrete transform.

- CWT requires infinite inputs, therefore is not convenient for computer analysis [3].

- The information provided by CWT is highly redundant.

- Decomposition or reconstruction with CWT requires a significant amount of computation time and resources. Therefore CWT is considerably sluggish to implement as compared to DWT.

The general equation of DWT for signal $x(k)$ is given by:

$$
D W T(m, n)=\frac{1}{\sqrt{c_{0}^{m}}} \sum_{k} x(k) \varphi\left(\frac{n-k d_{0} c_{0}^{m}}{c_{0}^{m}}\right)
$$


where $c_{0}$ and $d_{0}$ are discrete scaling and discrete translation factors respectively. Having fixed constant values generally, $c_{0}=2$ and $d_{0}=1$, these parameters, $c_{0}^{m}$ and $k d_{0} c_{0}^{m}$, are taken as constants. $m$ and $n$ are integers, representing frequency localization and time localization, correspondingly. The parameter $c_{0}^{m}$ produces the oscillatory frequency and length of the wavelet, whereas $k d_{0} c_{0}^{m}$ credits shifting (translation) position.

The idea for DWT computation is same as it is in CWT. In CWT there is a correlation among a wavelet at different scales. The given signal is calculated by varying the analysis window scale and shifting the window in time. It is multiplied by the given signal and then integrating over all times, as depicted in Figure 3. Whereas in discrete WT, signal $x$ is passed through series of digital high pass (HP) filters, to analyze high frequencies and through digital low pass (LP) filters, to analyze low frequencies. These filters are at different cut-off frequencies to evaluate signals at different scales. In this whole process the resolution of the signal is changed by filtering and the scale is changed by sampling operations [4-6].

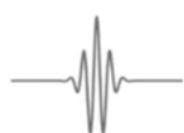

(a)

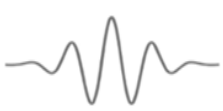

(b)

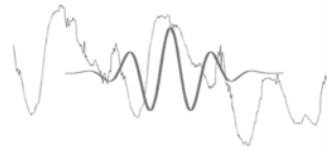

(c)
Fig. 3. (a) Small scale factor and high frequency wavelet. (b) Large scale factor and high frequency wavelet. (c) General graphic representation of CWT process where short duration wavelets are dilated and shifted to vary the timefrequency resolution.

\section{MULTI RESOLUTION ANALYSIS}

Multi resolution analysis (MRA) was developed as the basic frame work for wavelet expansion algorithm. With MRA, disturbance waveform decomposition and reconstruction can be obtained at various resolution levels and scales of short waveforms called mother wavelets. MRA is also termed as pyramidal coding which is similar to sub band coding method. In multi-level resolution analysis, decomposition is repeated up to several levels to increase frequency resolution for detail and approximation coefficients. With DWT-MRA, the decomposed signal can also be reconstructed through up sampling process to recover original time domain signals without losing any information. In MRA based wave decomposition, the sample waveform being analyzed is passed through half band low pass filter $g(k)$, which has impulse response $g$. This causes the convolution in discrete time, which can be mathematically defined as:

$$
(x * g)(n)=\sum_{k=-\infty}^{\infty} x(k) g(n-k)
$$

Likewise, the signal is also decomposed concurrently through half band HP filter $h(k)$. For the first level of decomposition i.e. down sampling by a factor of 2 , the outputs of the HP and LP filters are referred as detail D1 and approximation level A1 respectively. For level 2 decomposition the obtained approximation A1 coefficients are passed through the same HP and LP filters to produce coefficients A2 and D2 respectively. In the same way A2 coefficients are again passed through filters of same cut off frequency limits and so on. In this way down sampling is applied for further levels of wave decomposition. This process is largely termed as multi-level decomposition [7]. The filter output relations are mathematically expressed as:

$$
\begin{aligned}
& \varphi(n)=\sqrt{2} \sum_{k} g(n) \varphi(2 n-k) \\
& \varphi(n)=\sqrt{2} \sum_{k} h(n) \varphi(2 n-k)
\end{aligned}
$$

In (4) and (5), $k$ represents the number of samples. DWTMRA based decomposition is according to the Nyquist rule. Half the frequencies of the signal have now been removed and half the samples are discarded (Figure 4).

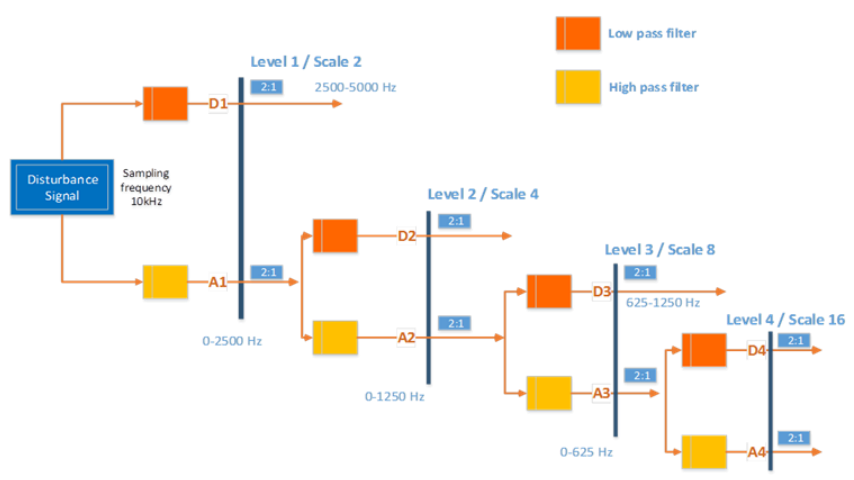

Fig. 4. DWT decomposition schematic diagram

Similarly with decomposition process the frequency band i.e. half of each filter output characterizes the signal. Therefore time resolution is reduced by a factor of 2 . Now the frequency resolution is doubled for each next level of decomposition. This is due to half input frequency band of the previous level and so on [5, 8-10]. The mathematical expressions for the approximation $A_{j}$ and detail level $D_{j}$ coefficients are given as:

$$
\begin{aligned}
& A_{j+1}(n)=\sum_{k} h(k-2 n) A_{j}(k) \\
& D_{j+1}(n)=\sum_{k} g(k-2 n) A_{j}(k)
\end{aligned}
$$

The mathematical relation for signal $f(n)$ expanded in relation to its orthogonal basis of scaling and wavelet function is shown in (8). Whereas (8) is basically characterized by one set of scaling coefficient, and one or several sets of wavelet coefficients:

$$
f(n)=\sum_{k} A_{1}(k) \emptyset(n-k)+\sum_{k} \sum_{j=1} D_{j}(n) 2^{-\frac{1}{2}} \varphi\left(2^{j} n-k\right)
$$

In (6)-(8), $j=1,2,3 \ldots$ represents the level of decompositions.

\section{DISCUSSION AND METHODOLOGY}

Real PQ waveforms frequently exhibit slow or abrupt variations, often punctuated with oscillations, harmonics, transients or other disturbances. These changes are the important part of the data, both perceptually and in terms of information of abnormality they provide. For the detection of disturbances, PQDs data can be obtained through real time PQ loggers, but disturbances generated using parametric equations (Table I), are practically more suitable and convenient [22]. 
TABLE I. PARAMETRIC EQUATIONS FOR COMMON POWER QUALITY DISTURBANCES [33]

\begin{tabular}{|c|c|c|c|}
\hline Sr.\# & PQ Disturbances & Mathematical Equations & Parameters \\
\hline 02. & Sag & $y(t)=A_{m}\left[1-\alpha\left(u\left(t-t_{1}\right)-u\left(t-t_{2}\right)\right)\right] \sin (\omega t)$ & $\begin{array}{c}0.1 \leq \alpha \leq 0.9 \\
T \leq t_{2}-t_{1} \leq 7 T\end{array}$ \\
\hline 03. & Swell & $y(t)=A_{m}\left[1+\alpha\left(u\left(t-t_{1}\right)-u\left(t-t_{2}\right)\right)\right] \sin (\omega t)$ & $\begin{array}{r}0.1 \leq \alpha \leq 0.8 \\
T \leq t_{2}-t_{1} \leq 7 T\end{array}$ \\
\hline 04. & Interruption & $y(t)=A_{m}\left[1-\alpha\left(u\left(t-t_{1}\right)-u\left(t-t_{2}\right)\right)\right] \sin (\omega t)$ & $\begin{array}{c}0.9 \leq \alpha \leq 1 \\
T \leq t_{2}-t_{1} \leq 7 T\end{array}$ \\
\hline 05. & Harmonics & $y(t)=A_{m}\left[\alpha_{1} \sin (\omega t)+\alpha_{3} \sin (3 \omega t)+\alpha_{5} \sin (5 \omega t)+\alpha_{7} \sin (7 \omega t)\right]$ & $\begin{array}{c}0.05 \leq \alpha_{3} \alpha_{5} \alpha_{7} \leq 0.15 \\
\sum \alpha_{i}^{2}=1\end{array}$ \\
\hline 06. & Flicker & $y(t)=A_{m}\left[1+\alpha_{f} \sin (\beta \omega t)\right] \sin (\omega t)$ & $\begin{array}{c}0.1 \leq \alpha_{f} \leq 0.2 \\
5 \leq \beta \leq 20 \mathrm{~Hz}\end{array}$ \\
\hline 07. & Oscillatory transients & $y(t)=A_{m}\left[\sin (\omega t)+\alpha^{-c\left(t-t_{1}\right) / \tau} \sin \omega_{n}\left(t-t_{1}\right)\left(u\left(t_{2}\right)-u\left(t_{1}\right)\right]\right.$ & $\begin{array}{c}0.1 \leq \alpha \leq 0.8 \\
0.5 T \leq t_{2}-t_{1} \leq 3 T \\
8 m s \leq \tau \leq 40 \mathrm{~ms} \\
300 \leq f_{n} \leq 900 \mathrm{~Hz}\end{array}$ \\
\hline 08. & Impulsive transients & $y(t)=A_{m}\left[1-\alpha\left\{u\left(t-t_{1}\right)-u\left(t-t_{2}\right)\right\}\right] \sin (\omega t)$ & $\begin{aligned} 0 & \leq \alpha \leq 0.414 \\
T / 20 & \leq t_{2}-t_{1} \leq T / 10\end{aligned}$ \\
\hline 10. & Harmonics with swells & $\begin{array}{l}y(t)=A_{m}\left[1+\alpha\left(u\left(t-t_{1}\right)-u\left(t-t_{2}\right)\right)\right] \\
{\left[\alpha_{1} \sin (\omega t)+\alpha_{3} \sin (3 \omega t)+\alpha_{5} \sin (5 \omega t)\right]}\end{array}$ & $\begin{array}{c}0.05 \leq \alpha_{3}, \alpha_{5}, \alpha_{7} \leq 0.15 \\
\sum \alpha_{i}^{2}=1\end{array}$ \\
\hline
\end{tabular}

Ten types of frequently occurred disturbances including both single and multiple disturbances are generated and simulated in Matlab. A function and structure is developed to produce random samples of each selected disturbance type. Where disturbance magnitude and duration over cycles can be changed in a wide range and controlled manner conferring to disturbance type and model equation parameters. For sample generation such parameters are randomly varied in compliance with the categories and characteristics of the power system. Moreover, noise of any level in $\mathrm{dB}$ can be added to the simulated disturbances so that the real PQD phenomenon can be reflected with the simulated waveforms. In this work, waveforms of pure voltage sine wave, sags, swells, interruptions, harmonics, impulsive transients, oscillatory transients, sags with harmonics and swells with harmonics are considered for generation. One hundred random sample cases with 10 cycles at $50 \mathrm{~Hz}(0.2 \mathrm{~s})$ for each disturbance were produced. The sampling frequency was $10 \mathrm{kHz}$. Both magnitudes as well as time of event occurrence were diverse in accordance with the aforementioned standard. All disturbance samples, carrying random variations and disturbances at unsystematic time instants were passed through series of HP and LP filters (Figure 4) to get approximateion $A_{j}$ and detail coefficient $D_{j}$, waveforms. With full power and efficient time frequency localization characteristics embedded in MRADWT, all disturbance samples were tested and utilized for detection as a visualized scheme from the number of approximations and detail levels of each disturbance. The number of such coefficient waveforms not only detects abnormalities but also serves the cause of feature extraction process. For machine learning based recognition and classification applications in PQ, feature extraction process is considered the most vital process. Extracted features are typically the measured data, normally obtained from approximation $A_{j}$ and detail coefficients $D_{j}$ waveforms. This feature extraction data may serve as training and testing data set for machine learning classifier algorithms. More coefficient waveforms i.e. decomposition level outputs, result to better feature vector hence may improve classification accuracy.

\section{SIMULATION RESULTS}

PQD patterns are produced using model parametric equations (Figures 5-6). Disturbances with added noise of 20dB SNR are also generated, depicted in Figure 5 to mimic real PQ noisy waveforms.
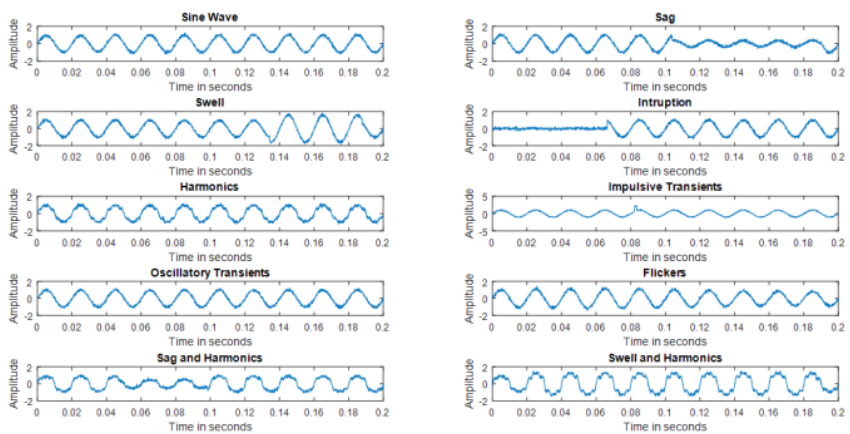

Fig. 5. Noise riding power quality disturbances with 20dB SNR

One hundred samples of each disturbance were generated. For all these samples of 10 generated disturbances, time frequency localization of each disturbance event is achieved and succeeded by the DWT-MRA technique in Matlab/Workspace environment (Figure 6). 


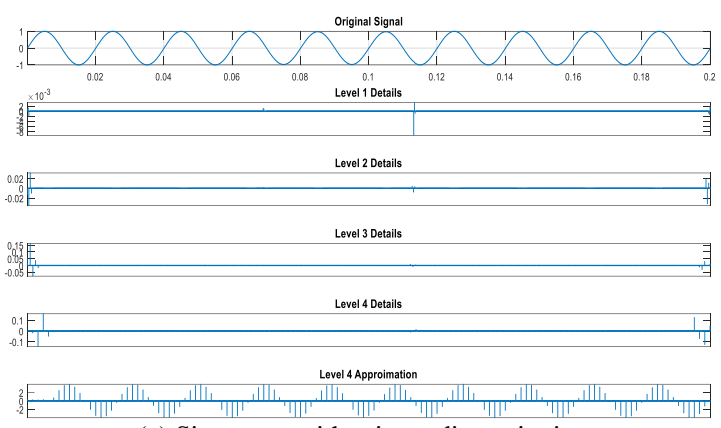

(a) Sine wave with minute discontinuity

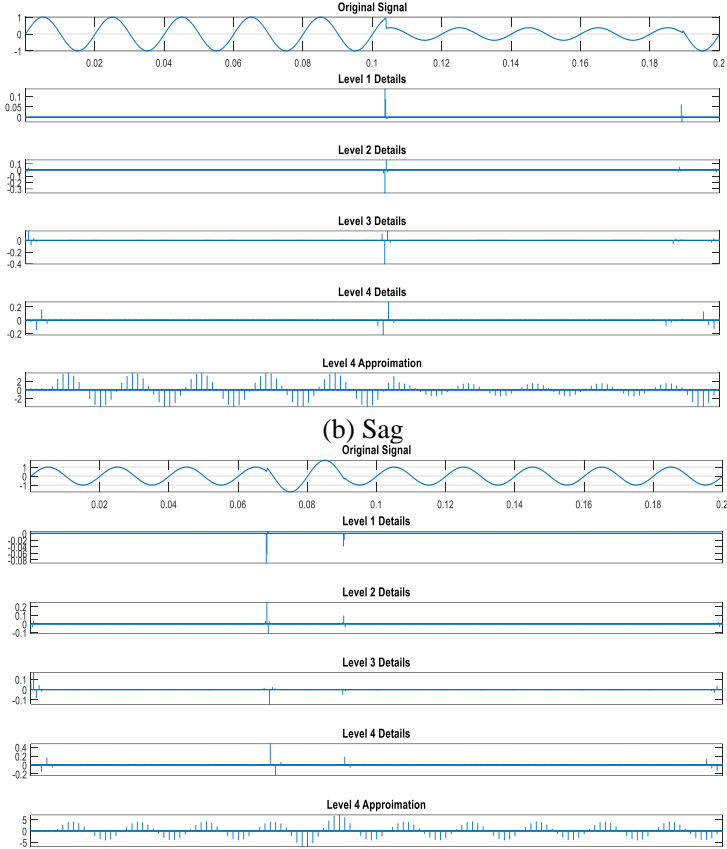

(c) Swell

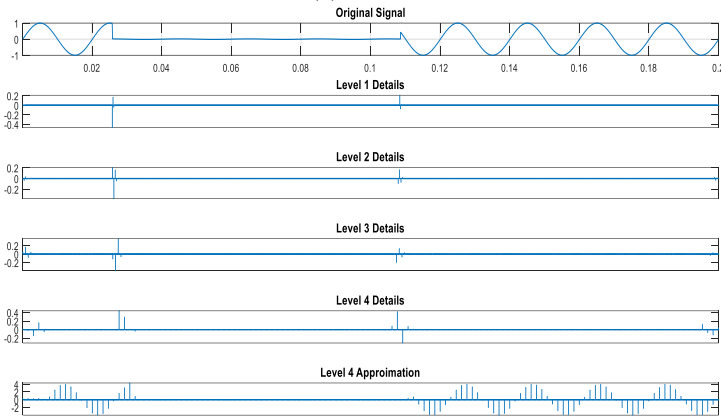

(d) Interruption

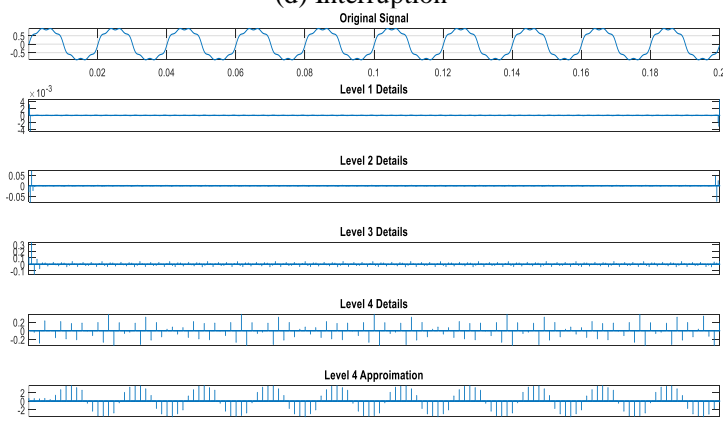

(e) Harmonics

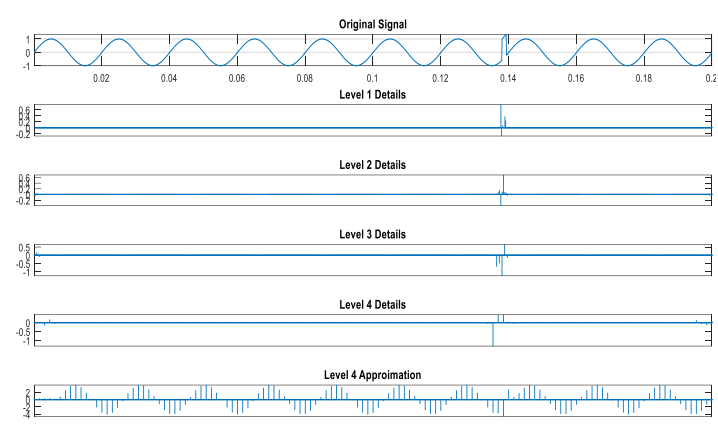

(f) Impulsive transient

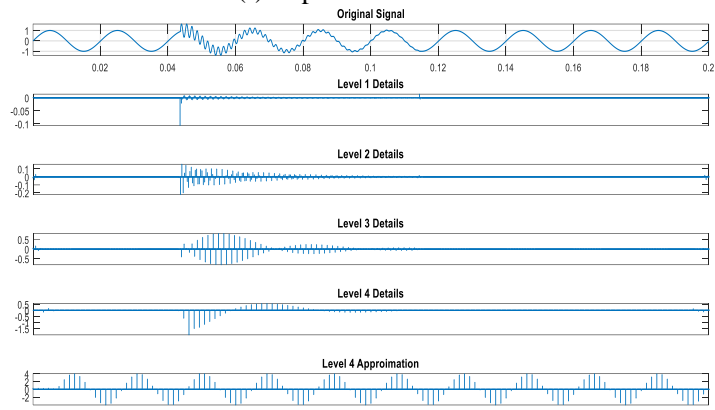

(g) Oscillatory Transient

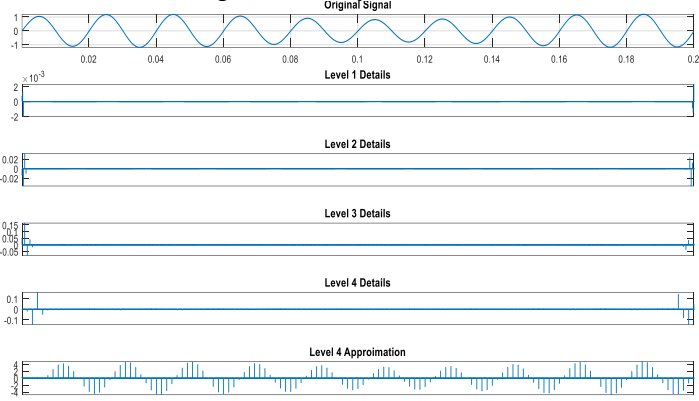

(h) Flicker

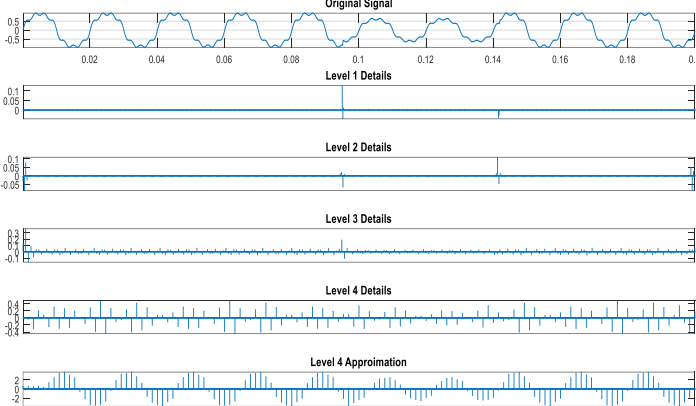

(i) Sag with harmonics

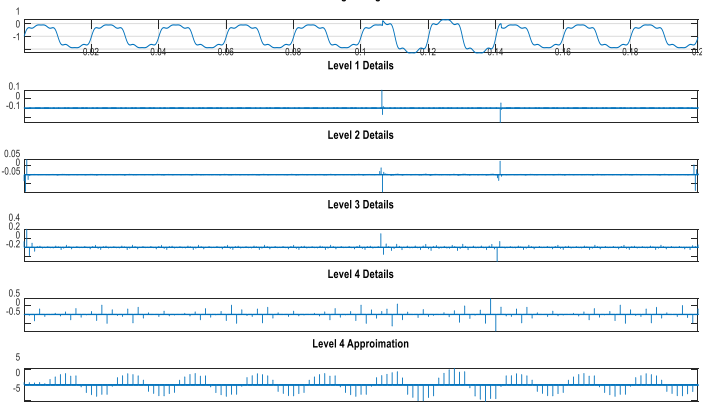

(j) Swell with harmonics

Fig. 6. (a) to (j) Generated disturbances with discretized detail level 1-4 and level 4 approximation coefficient waveforms. 
Only 1 sample of each disturbance is shown in Figure 6(a)(j) which is decomposed by Daubechies db4 wavelet filter for disturbance detection in time frequency domain. Each outcome in Figure 6 consists of 6 waveforms, where the first waveform is the originally generated sample of each mentioned disturbance. The next 4 waveforms show discretized 1-4 detail levels, having high frequency, while the sixth waveform shows the discretized 4th level approximation coefficients, devising the fundamental frequency components. Each Figure is correspondingly obtained for event detection in a visualized scheme, but such detail and approximation coefficient waveforms are also helpful for machine learning based automatic recognition and classification of disturbances. For additional validation of results a power system model in Matlab/Simulink can also be developed by simulating faults, VAR compensation etc. Several numerical approaches will be implemented to analyze the severity and typical effects of disturbances. As per simulation results the micro controller or a FPGA prototype hardware will be developed and with details to be published in a future extended version.

\section{CONCLUSION}

This paper presents a power quality monitoring phenomenon, including, causes, issues and classification. Wavelet transforms and types were also discussed. Individual and multiple disturbance samples of varying time, normalized magnitude, with and without noise were generated in Matlab to simulate PQD events based on IEEE STD-1159-2009. The simulation results show that the generated disturbances are analogous to real PQDs. The simulation results also showed that the application of DWT-MRA to generated disturbances is so powerful that even a slight discontinuity/disturbance and event duration is localized accurately at any instant in timefrequency plane. The data obtained comprising approximation and detail levels can be executed in machine learning based automatic classification of PQDs using various recognition and classification algorithms. The methodology can also open new possibilities and techniques in automatic clearance or anticipation of minor and major abnormal operations in power systems.

\section{REFERENCES}

[1] K. Thirumala, M. S. Prasad, T. Jain, A. C. Umarikar, "Tunable-Q wavelet transform and dual multiclass SVM for online automatic detection of power quality disturbances", IEEE Transactions on Smart Grid, Vol. 9, No. 4, pp. 3018-3028, 2018

[2] H. Liu, F. Hussain, Y. Shen, S. Arif, A. Nazir, M. Abubakar, "Complex power quality disturbances classification via curvelet transform and deep learning”, Electric Power Systems Research, Vol. 163, pp. 1-9, 2018

[3] M. ElNozahy, R. El-Shatshat, M. Salama, "Single-phasing detection and classification in distribution systems with a high penetration of distributed generation", Electric Power Systems Research, Vol. 131, pp. 41-48, 2016

[4] E. Fuchs, M. A. Masoum, Power Quality in Power Systems and Electrical Machines, Academic Press, 2011

[5] A. A. M. Z. Suhail Khokhar, M. A. Bhayo, A. S. Mokhtar, "Automated recognition of single \& hybrid power quality disturbances using wavelet transform based support vector machine", Jurnal Teknologi, Vol. 79, No. 1, pp. $97-105,2017$

[6] S. Chakraborty, A. Chatterjee, S. K. Goswami, "A dual-tree complex wavelet transform-based approach for recognition of power system transients", Expert Systems, Vol. 32, No. 1, pp. 132-140, 2015
[7] S. R. Mohanty, N. Kishor, P. K. Ray, J. P. S. Catalao, "Comparative Study of Advanced Signal Processing Techniques for Islanding Detection in a Hybrid Distributed Generation System", IEEE Transactions on Sustainable Energy, Vol. 6, No. 1, pp. 122-131, 2015

[8] S. Khokhar, A. A. B. M. Zin, A. S. B. Mokhtar, M. Pesaran, "A comprehensive overview on signal processing and artificial intelligence techniques applications in classification of power quality disturbances", Renewable and Sustainable Energy Reviews, Vol. 51, pp. 1650-1663, 2015

[9] R. Kumar, B. Singh, D. T. Shahani, "Recognition of Single-stage and Multiple Power Quality Events Using Hilbert-Huang Transform and Probabilistic Neural Network", Electric Power Components and Systems, Vol. 43, No. 6, pp. 607-619, 2015

[10] L. C. M. Andrade, M. Oleskovicz, R. A. S. Fernandes, "Adaptive threshold based on wavelet transform applied to the segmentation of single and combined power quality disturbances", Applied Soft Computing, Vol. 38, pp. 967-977, 2015

[11] A. Domijan, G. T. Heydt, A. P. S. Meliopoulos, S. S. Venkata, S. West, "Directions of research on electric power quality", IEEE Transactions on Power Delivery, Vol. 8, No. 1, pp. 429-436, 1993

[12] R. Kumar, B. Singh, D. Shahani, C. Jain, "Dual-Tree Complex Wavelet Transform-Based Control Algorithm for Power Quality Improvement in a Distribution System", IEEE Transactions on Industrial Electronics, Vol. 64, No. 1, pp. 764-772, 2017

[13] S. Salcedo-Sanz, J. L. Rojo-Alvarez, M. Martinez-Ramon, G. CampsValls, "Support vector machines in engineering: An overview", Wiley Interdisciplinary Reviews: Data Mining and Knowledge Discovery, Vol. 4, No. 3, pp. 234-267, 2014

[14] F. A. Borges, R. A. Fernandes, I. N. Silva, C. B. Silva, "Feature extraction and power quality disturbances classification using smart meters signals", IEEE Transactions on Industrial Informatics, Vol. 12, No. 2, pp. 824-833, 2016

[15] Z. Liu, Q. Hu, Y. Cui, Q. Zhang, "A new detection approach of transient disturbances combining wavelet packet and Tsallis entropy", Neurocomputing, Vol. 142, pp. 393-407, 2014

[16] S. Khokhar, A. A. B. Mohd Zin, A. S. B. Mokhtar, M. Pesaran, "A comprehensive overview on signal processing and artificial intelligence techniques applications in classification of power quality disturbances", Renewable and Sustainable Energy Reviews, Vol. 51, pp. 1650-1663, 2015

[17] M. Biswal, P. K. Dash, "Measurement and Classification of Simultaneous Power Signal Patterns With an S-Transform Variant and Fuzzy Decision Tree", IEEE Transactions on Industrial Informatics, Vol. 9, No. 4, pp. 1819-1827, 2013

[18] S. Khokhar, A. A. Mohd Zin, A. P. Memon, A. S. Mokhtar, "A new optimal feature selection algorithm for classification of power quality disturbances using discrete wavelet transform and probabilistic neural network", Measurement, Vol. 95, pp. 246-259, 2017

[19] J. Barros, R. I. Diego, M. de Apraiz, "Applications of wavelets in electric power quality: Voltage events", Electric Power Systems Research, Vol. 88, pp. 130-136, 2012

[20] M. Tuljapurkar, A. A. Dharme, "Wavelet based signal processing technique for classification of power quality disturbances", 5th International Conference on Signal and Image Processing, Bangalore, India, South Korea, January 8-10, 2014

[21] A. A. Abdoos, Z. Moravej, M. Pazoki, "A hybrid method based on time frequency analysis and artificial intelligence for classification of power quality events", Journal of Intelligent \& Fuzzy Systems, Vol. 28, No. 3, pp. 1183-1193, 2015

[22] A. A. Abdoos, P. K. Mianaei, M. R. Ghadikolaei, "Combined VMDSVM based feature selection method for classification of power quality events", Applied Soft Computing, Vol. 38, pp. 637-646, 2016 